\title{
The utilization of landscape pictures extracted from open picture collections for the determination of interest in spatial features
}

\author{
Jens Ingensand ${ }^{1}$, Jean-Christophe Foltête ${ }^{2}$, Stéphane Cretegny ${ }^{1}$, Nicolas \\ Blanc $^{1}$, and Sarah Composto ${ }^{1}$
${ }^{1}$ University of Applied Sciences Western Switzerland (HES-SO)
${ }^{2}$ University of Burgundy - Franche-Comté \\ Corresponding author: \\ Jens Ingensand ${ }^{1}$ \\ Email address: jens.ingensand@heig-vd.ch
}

\section{ABSTRACT}

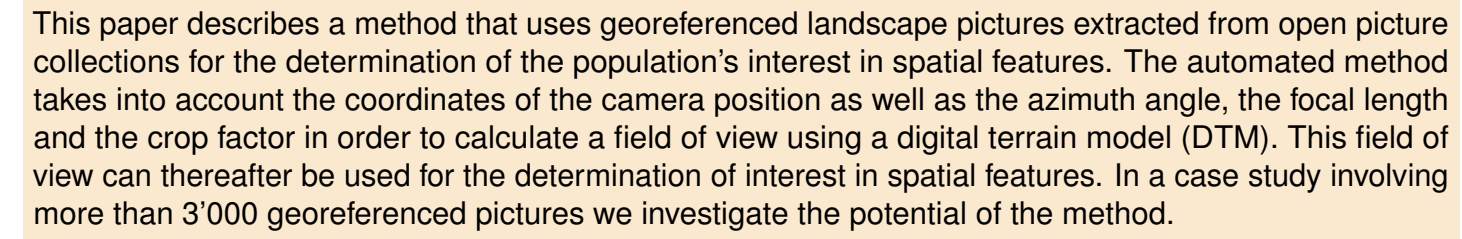

This paper describes a method that uses georeferenced landscape pictures extracted from open picture collections for the determination of the population's interest in spatial features. The automated method takes into account the coordinates of the camera position as well as the azimuth angle, the focal length and the crop factor in order to calculate a field of view using a digital terrain model (DTM). This field of view can thereafter be used for the determination of interest in spatial features. In a case study involving more than 3'000 georeferenced pictures we investigate the potential of the method.

\section{INTRODUCTION}

Pictures taken by a smartphone or a sensor-equipped tablet computer usually keep the sensor information and other device-related specifications in the EXIF-tags. When these photos are uploaded to photo sharing sites and applications such as Instagram (https://www.instagram.com/), Google Photos (https://photos.google.com/) or flickR (https://flickr.com) these tags are shared along with the pictures.

Today these picture collections contain vast quantities of photos. Many collections offer the possibility to access and download the pictures along with their metadata using an API, allowing a user to e.g. extract pictures for a specific region or a specific period of time.

Several studies have used public picture collections in the field of landscape perception and classification analysis: Dunkel (2015); Crandall et al. (2009); Chen et al. (2018) for instance have used the tags that a user can add to pictures along with the coordinates to classify the landscape and to create maps that show the prominence of tags. Tenerelli et al. (2017) have used pictures extracted from flickR to asses the relationship between a landscape's visual character and scenic beauty. In their approach the authors used viewsheds to determine which visual indicator can be used to classify pictures. Seresinhe et al. (2018) have combined public pictures extracted for the United Kingdom with OpenStreetMap data for the determination of the "scenicness" of the pictures. In their approach the authors excluded for instance pictures taken within buildings footprints to only keep landscape pictures.

\section{UTILIZATION OF THE FIELD OF VIEW EXTRACTED FROM PUBLIC PIC- TURE COLLECTIONS}

The utility of GPS coordinates extracted from photo collections for research purposes has been demonstrated throughout several studies. The EXIF tags however contain additional parameters that allow us to further refine the position: the azimuth parameter (i.e the direction of the mobile device at the time a picture has been taken), the crop factor and the focal length parameters allow us to determine a field of view from the point where a picture has been taken. 
The determination of the field of view using these parameters relies on two assumptions:

1. Since the angles roll and pitch are not included in the EXIF tags of the picture, we must assume that the user has kept the device straight while taking a picture.

2. The azimuth angle needs to be accurate for the determination of the field of view.

The accuracy of the azimuth angle parameter (which indicates the direction of the mobile device at the time a picture has been taken) is more difficult to asses. In order to address this parameter's accuracy we extracted 29 photos that had been taken in the Swiss village of Zermatt. All photos show the well-known Matterhorn which is situated at a distance of 10 kilometers from Zermatt. With these photos we were able to determine that $70 \%$ of the photos had angles that matched the calculated azimuth angle between the camera position and the mountain top. Figure 1 illustrates the process. An important finding was that the accuracy appeared to increase with the recentness of the mobile device.

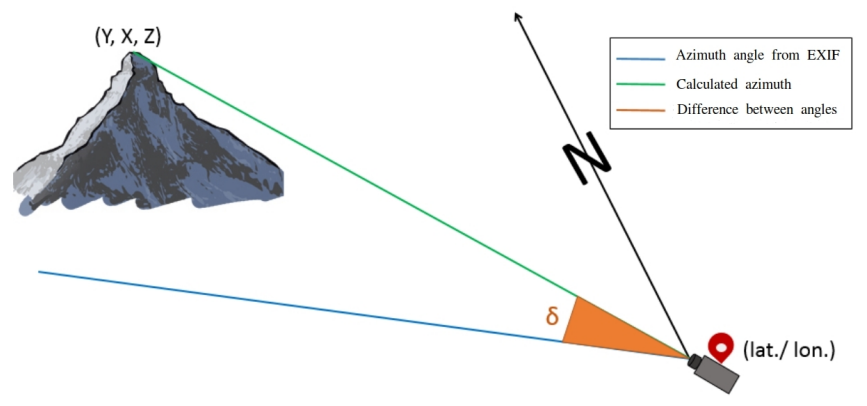

Figure 1. Validation process of the azimuth angles

Due to the fact that a mobile device often has several cameras and can be held in different positions (landscape - portrait; front facing camera, rear-facing camera) the azimuth angle often needs to be corrected (e.g. -180 degrees). This correction can be computed depending on other EXIF-parameters.

With the location information stored in the photos (azimuth angle, GPS coordinates of the camera position) as well as the important camera parameters focal length and crop factor we can compute a 3D field of view using a digital terrain model (DTM). This process follows three steps:

1. Around a GPS point extracted from each photo, the viewshed is created using the open-source GIS SAGA and a digital terrain model (DTM)

2. The opening angle is calculated using the azimuth angle, the crop factor and the focal length.

3. The viewshed is vectorized and cut with the opening angle.

This 3D field of view can thereafter be matched with other reference data (e.g. land-use layers and points of interest) to classify the scene.

\section{CASE STUDY}

For our case study we have downloaded 5'131 images for the Swiss Canton of Vaud. We filtered the images using their position within the administrative boundaries and the building footprints from OpenStreetMap, (www.openstreetmap.org), with the goal to only keep pictures that had been taken outside. The result were 3' 483 potential landscape images. For each picture we calculated the field of view using the aforementioned method.

The overlay of all polygons generated for all pictures allowed us to generate different results such as maps that show areas which are visible in a determined number of pictures. Figure 2 represents a map with the accumulated fields of view.

The first results of our case study show that a vast majority of pictures were taken from points that are easily accessible (e.g roads, paths, railroads, etc). Waterbodies such as lake Geneva are very prominent features as well as adjacent mountain ranges. One explanation to this result is the topographical openness 


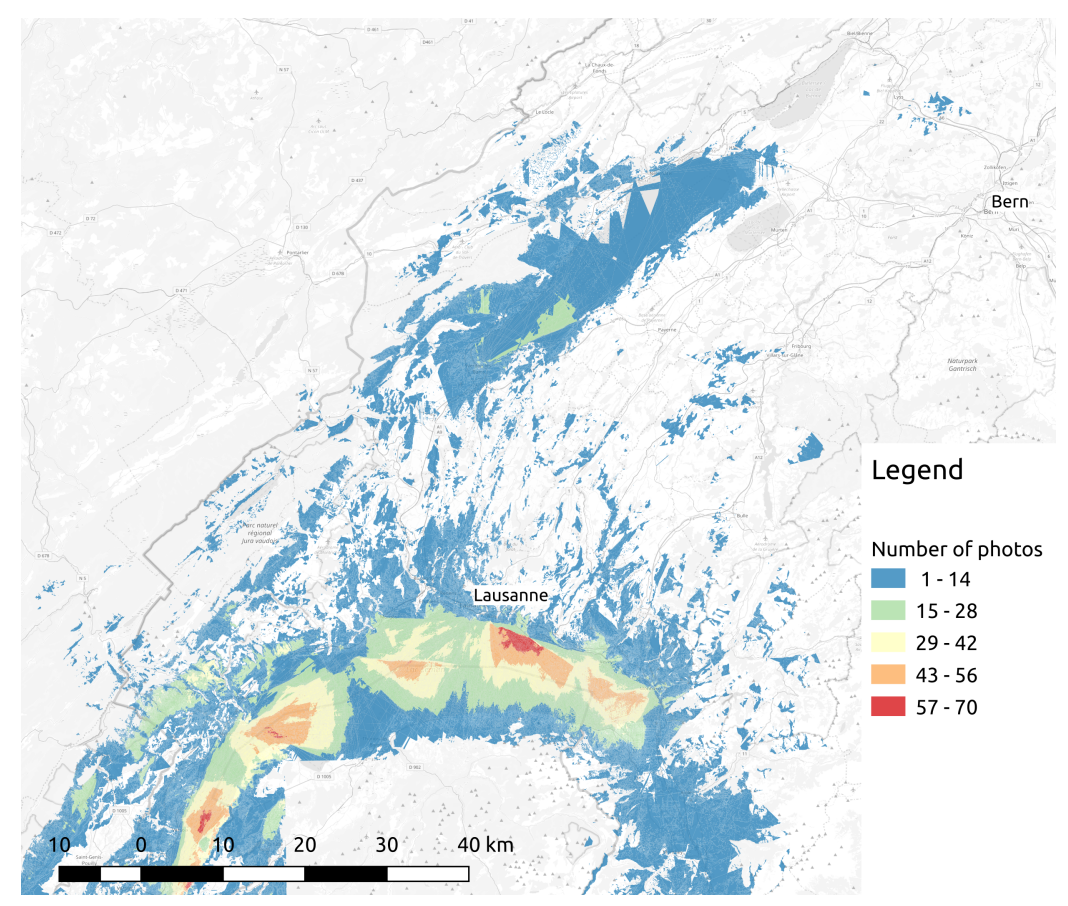

Figure 2. Accumulated fields of view calculated for landscape photos taken from a point within the canton of Vaud

of waterbodies (no obstacles) another interpretation is the presence of touristic spots near the lakes such as the Lavaux Region (UNESCO World heritage site) east of Lausanne. Rural areas on the other hand are much less prominent than areas around populated regions such as the shores of lake Geneva and Lake Neuchâtel. These findings lead us to the conclusion that population density and the accessibility of places need to be considered as well in the evaluation of the population's interest in spatial features.

\section{CONCLUSIONS AND PERSPECTIVES}

The described method has a high potential for further exploration. One point that we are addressing at the time of writing is the influence of a point's accessibility (e.g. if a point is situated on a road and thus easy to access) and the bias that certain regions are more or less populated. We have therefore developed an algorithm that places random points on roads, railroads, and footpaths, and that calculates the viewsheds based on these points. The goal is then to substract the fields of view that have been taken in pictures from the accumulated viewsheds that are potentially visible. The resulting maps will allows us refine the interpretation of spatial features that are of interest.

Another aspect that we want to analyze is the automated characterization of photos and their content using machine-learning algorithms. Many researchers such as Dunkel (2015); Chen et al. (2018) have used image tags added by users which are subjective elements. Machine learning algorithms would allow for a more objective and uniform characterization.

The resulting maps that we have generated can be used by land planners as a basis for spatial decision making; e.g. for the installation of windmills in areas that are not visible in any photo or for the establishment of touristic footpaths or even for planned construction work.

The utilization of open picture collections and spatial knowledge that can be extracted from such data is an interesting field of investigation since it allows researchers to use subjective data in an objective way.

\section{REFERENCES}

Chen, Y., Parkins, J. R., and Sherren, K. (2018). Using geo-tagged instagram posts to reveal landscape values around current and proposed hydroelectric dams and their reservoirs. Landscape and Urban Planning, 170:283 - 292. 
Crandall, D. J., Backstrom, L., Huttenlocher, D., and Kleinberg, J. (2009). Mapping the world's photos. In Proceedings of the 18th International Conference on World Wide Web, WWW '09, pages 761-770, New York, NY, USA. ACM.

Dunkel, A. (2015). Visualizing the perceived environment using crowdsourced photo geodata. Landscape and Urban Planning, 142:173 - 186. Special Issue: Critical Approaches to Landscape Visualization.

Seresinhe, C. I., Moat, H. S., and Preis, T. (2018). Quantifying scenic areas using crowdsourced data. Environment and Planning B: Urban Analytics and City Science, 45(3):567-582.

Tenerelli, P., Puffel, C., and Luque, S. (2017). Spatial assessment of aesthetic services in a complex mountain region: combining visual landscape properties with crowdsourced geographic information. Landscape Ecology, (32):1097-1115. 